\title{
Déploiement coopératif d'un dictionnaire électronique de données administratives
}

\author{
Isabelle Boydens*** \\ * Université Libre de Bruxelles \\ Faculté de Philosophie et Lettres \\ Section Sciences de l'information et de la documentation \\ CP 175/02 \\ Avenue FD Roosevelt, 50, B. 1050 Bruxelles, Belgique \\ iboydens@ulb.ac.be \\ ** SmalS-MvM \\ Unité d'exploitation Développement des applications \& consultance \\ Section Recherche \\ Rue du Prince Royal, 102, B. 1050 Bruxelles, Belgique \\ isabelle.boydens@smals-mvm.be
}

RÉSUMÉ. L'interprétation de l'information administrative est un processus complexe : en effet, la signification des concepts représentés est empirique et évolutive. Les incidences sociales de ce processus sont toutefois cruciales : ainsi, les montants traités via les bases de données de la sécurité sociale belge s'élèvent annuellement à environ 35 milliards d'euros. Cet article propose un ensemble de recommandations conceptuelles issues du déploiement coopératif d'un dictionnaire électronique destiné à faciliter l'interprétation et la gestion de ces bases de données dans un environnement multilingue: traitement de l'information structurée, non structurée et semi-structurée, mise en place de procédures d'héritage partiel, de tables de paramètres homogènes, de mécanismes de gestion des versions et, enfin, d'un système de workflow en vue de la validation des définitions.

ABSTRACT. Administrative information interpretation is a complex process because of the empirical and changing meaning of the concepts. However, the social consequences of this process are crucial : contributions collected by way of the Belgian social security databases amount each year to approximately 35 billions euros. This paper presents conceptual recommandations based on the conception of an electronic dictionary intended to improve the databases interpretation and management in a multilingual environment : structured, non structured and semi-structured information management, partial inheritance procedures handling, homogeneous parameter tables management, version modeling and workflow development in order to insure the validation of the definitions.

MoTS-CLÉS: herméneutique, collecticiels, workflow, métadonnées gestion des versions, système documentaire multilingue, intégration des données.

KEYWORDS: hermeneutics, groupware, workflow, metadata, version modeling, multilingual documentary system, data integration.

Document numérique. Volume $5-n^{\circ} 3-4 / 2001$, pages 27 à 43 


\section{Introduction}

Au sein d'un système d'information, les données ne sont jamais « données » dans une évidence intuitive. Les données se construisent progressivement (Boydens, 1999). L'étude conceptuelle de ce processus de construction est cruciale sur le plan opératoire lorsque l'informatique requiert un agencement étroit entre l'homme et la machine. Ainsi est-ce le cas des applications coopératives de type groupwareworkflow.

Dans cet article, nous évoquons cette question à travers l'étude d'une application coopérative déployée dans le cadre des projets d'e-governement de l'administration fédérale belge. Il s'agit d'un dictionnaire électronique de données administratives destiné à documenter les bases de données de la sécurité sociale au fil de leurs versions successives. Ces ensembles informatiques permettent le prélèvement des cotisations ainsi que leur redistribution aux assurés sociaux, ce qui implique le traitement annuel d'environ 35 milliards d'euros.

L'application vise à automatiser partiellement les procédures de saisie, de traduction et de validation de la documentation, à en renforcer l'intégrité et à suivre aisément l'évolution des mises à jour des bases de données correspondantes. Ce dictionnaire électronique est accessible en consultation (partie front end) sur le portail de la sécurité sociale belge (http://www.socialsecurity.be) ${ }^{1}$ et a été développé via un système de groupware-workflow. Nous évaluerons plus loin plusieurs apports et limites de cet environnement de développement. Sur le plan conceptuel, nous présenterons les éléments fondamentaux de l'application, tels que la mise en œuvre, dans un environnement documentaire multilingue, d'une structure d'héritage partiel couplée à un mécanisme de gestion de versions.

Les fonctionnalités coopératives de l'application documentaire répondent à trois exigences pratiques. En premier lieu, les définitions administratives se construisent progressivement en fonction de l'interprétation, souvent complexe, de la législation et de la prise en compte des contraintes de l'informatique. Ce processus de construction requiert une coopération étroite entre divers acteurs issus de cultures diverses (juristes, fonctionnaires de l'administration, informaticiens, traducteurs, secrétaires). En second lieu, la documentation diffusée inclut des informations de nature hétérogène (données «structurées », champs libres ou encore, «objets » attachés issus d'environnements bureautiques) exigeant un outil fédérateur (Phelps et $a l$, 2000), de type «collecticiel ». En troisième lieu, il s'agit idéalement de développer un système de «méta-information » qui évolue de façon cohérente et synchrone avec les bases de données correspondantes : nous verrons que cet idéal n'est jamais atteint mais qu'il est possible d'y tendre.

1. L'application a été développée au sein de l'unité d'exploitation Développement des applications et consultance de la SmalS-MvM. Le lien suivant donne accès à une application de ce type en ce qui concerne la base de données DmfA (déclaration multifonctionnelle) : http://www.socialsecurity.be/portail/glossaires/dmfa.nsf/web/glossary_home_fr 
De la mise en œuvre pratique de ces trois contraintes se dégage un ensemble d'enseignements méthodologiques généralisables. Nous proposons en premier lieu une évaluation de l'environnement de l'application et de quelques questions méthodologiques que soulève sa mise en œuvre dans le contexte du «web sémantique » et des systèmes de travail coopératif (section 2). Nous examinons ensuite les points suivants, déployant l'analyse d'une perspective statique vers une perspective dynamique, caractéristique des applications coopératives : les arbitrages que requiert la gestion de l'information dite «structurée », «non» ou «semistructurée » (section 3), les systèmes de groupware-workflow en tant qu'instruments d'aide à l'interprétation (section 4), et, enfin, les applications coopératives en tant qu'instruments d'aide à la construction de l'information (section 5).

\section{Environnement de l'application et questions méthodologiques}

\subsection{De l'instabilité des définitions administratives}

Le développement d'un dictionnaire de données est crucial lorsque l'information à traiter est instable. Examinons le cas de l'information administrative où cette instabilité tient à deux facteurs. D'une part, les modifications de la législation, dont certaines ont un effet rétroactif (Thomasset et al., 1997), sont fréquentes et ont des incidences parfois spectaculaires ${ }^{2}$.

Cette instabilité tient, d'autre part, au caractère «empirique » de l'information administrative qui est toujours sujette à interprétation. Prenons un exemple. La législation sociale belge est variable selon qu'elle s'applique aux ouvriers ou aux employés, les premiers et les seconds se distinguant selon la nature prépondérante de leurs activités manuelles ou intellectuelles ${ }^{3}$.

Cependant, cette distinction n'est pas aisée à opérer dans la pratique, de nombreux métiers incluant à la fois des tâches manuelles et intellectuelles. Or, dans le cadre de la gestion informatique, il faut trancher : la donnée «code travailleur » reçoit une valeur parmi un nombre limité de codes autorisés. Par contre, dans le «réel observable», le statut d'un même individu peut fluctuer, en fonction des interprétations de la législation (comme en témoigne l'ampleur de la jurisprudence), d'une part, et de l'interprétation de la situation de fait (l'activité observée d'un individu), d'autre part.

2. Citons un exemple survenu au Royaume-Uni : en février 1998, le nombre de chômeurs a subitement augmenté de 500000 unités parce que le gouvernement de Tony Blair avait décidé de modifier la définition du concept de chômeur en y incluant les personnes sans emploi ne bénéficiant pas d'allocations de chômage.

3. Loi du 3 juillet 1978 relative aux contrats de travail. Moniteur belge, 22 août 1978. 
A l'intérieur de la base de données, les transformations observées se traduisent par des modifications discrètes et formellement identifiables. Une fois appréhendée par l'homme, la signification de l'information fluctue avec les arbitrages que requiert son interprétation (Bachelard, 1981).

La prise en compte de ces difficultés conceptuelles est fondamentale en vue d'interpréter les données administratives et de traiter le plus équitablement possible les dossiers de chaque citoyen. Dans une perspective opératoire, nous proposons de schématiser le processus de construction de l'information en vue de concevoir un système documentaire permettant d'en faciliter la gestion.

\subsection{Le processus de transformation de l'information}

Deux types d'événements peuvent être à l'origine de la création d'une nouvelle version documentaire (figure 1): une modification législative ou des difficultés d'interprétation. La structure de l'application coopérative correspondante devra épouser au mieux la dynamique de ces deux types d'événements.

Une modification législative (processus P1) implique une adaptation des directives administratives (processus P2) et ensuite une modification des directives techniques (processus P3), présidant à la programmation proprement dite de la base de données. Une fois ces adaptations effectuées, la documentation disponible (directives administratives, fichier D2 et techniques, fichier D3) est utilisée au sein de l'administration et diffusée aux citoyens, fournisseurs de l'information (processus P4).

Des difficultés d'interprétation peuvent apparaitre lors de l'adaptation de la base de données (Boucourechliev, 1992) ou encore du traitement et de l'interprétation des données envoyées par les employeurs (processus P5 et P6). Ces difficultés d'interprétation peuvent impliquer une nouvelle adaptation des directives administratives et techniques (Boydens et al., 1997). Par exemple, on observe, lors de l'interprétation d'une déclaration sociale, l'émergence d'un nouveau secteur d'activité non prévu initialement dans la législation et nécessitant un traitement inédit, ce qui implique une modification du schéma de la base de données.

Le fichier D3 de la figure 1 représente la documentation «cible » que nous proposons de traiter. A l'heure actuelle, l'application présentée ici permet le traitement de dizaines d'applications documentaires ${ }^{4}$ correspondant chacune à une base de données spécifique.

4. Individuellement, chaque base de données comporte de cent à trois cents champs dont la définition technique est spécifiée dans l'application documentaire. 


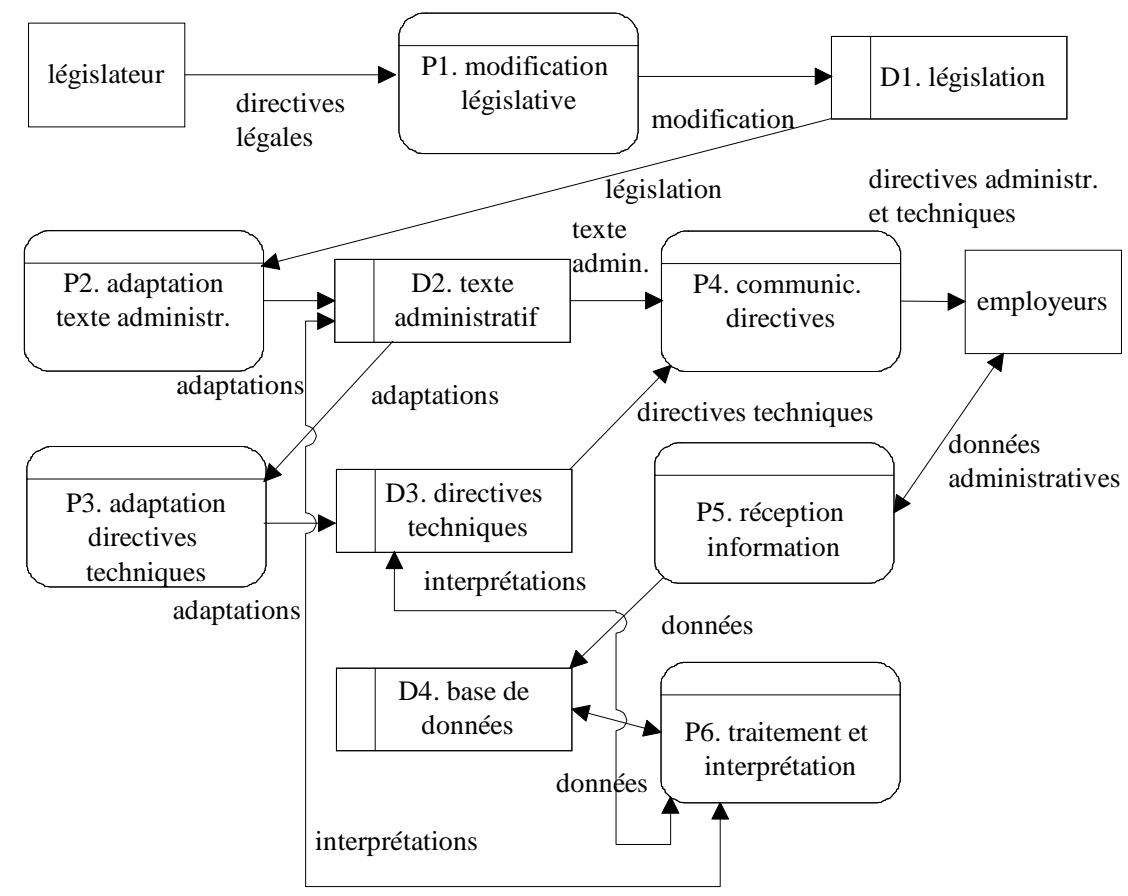

Figure 1. Processus de transformation de la documentation

\subsection{Le contexte du web sémantique et des systèmes de travail coopératif}

Dans le domaine du traitement de l'information, deux défis importants émergent actuellement : l'intégration d'applications hétérogènes, d'une part et la conception d'applications de type WOPM (Write Once Publish Many), d'autre part, en vue de diffuser un même contenu sous des formats différents, ce même contenu traversant des communautés distinctes d'auteurs et de collaborateurs. A l'échelle du web, le protocole WebDAV, World Wide Web Distributed Authoring and Versioning (Qu et al., 2000), ainsi que les systèmes de travail coopératif (CSCW systems) vont dans ce sens (Osguthorpe G. et al., 1996).

La problématique présentée dans cet article s'inscrit dans ce contexte car elle requiert la gestion de documents hétérogènes construits progressivement par différents acteurs et diffusés dans un environnement web. Afin de réduire la complexité sémantique de telles applications, la conception d'ontologies est préconisée dans la mouvance du web sémantique (Delteil et al., 2001, Mcllraith et al., 2001) : une ontologie peut s'apparenter à une collection de concepts génériques réutilisables permettant de décrire et de structurer un domaine d'application donné. Dans cet article, nous proposons un mode de représentation et d'organisation des 
concepts présidant à l'échange de la documentation électronique administrative, dans le cadre d'une «communauté virtuelle de travail ».

Ces développements soulèvent toutefois plusieurs questions critiques sur le plan méthodologique. Citons en deux. En premier lieu, de nombreuses méthodes de modélisation formelles, telles que préconisées dans le cadre du web sémantique (Gray, 2001), reposent sur les traditionnels schémas entités-associations (Chen, 1977) largement appliqués au monde des bases de données relationnelles depuis une vingtaine d'années. Mais lorsque l'on applique pratiquement ces modèles dans le cadre des collecticiels, une difficulté se présente : la structuration de l'information n'étant pas nécessairement relationnelle au sein de ces systèmes, les associations ou encore l'intégrité référentielle ne peuvent être représentées de façon orthodoxe. Il en découle une perte de précision lors du développement de l'application. En second lieu, le déploiement de telles applications coopératives soulève de vastes questions organisationnelles ${ }^{5}$. C'est la raison pour laquelle, comme nous le verrons au terme de cet article, la mise en production de telles applications ne peut aboutir que grâce à un investissement humain important en vue d'assurer une communication efficace entre les membres de l'espace virtuel de travail. En contrepartie de ces questions critiques, les applications concernées offrent une grande souplesse de travail. Nous reviendrons plus concrètement sur l'arbitrage entre rigueur formelle et flexibilité de développement dans les lignes qui suivent.

\section{De l'information « structurée », « non structurée » et « semi-structurée »}

On observe au sein de la documentation technique administrative la coexistence d'informations de nature hétérogène. Nous présentons ici les notions d'information structurée, non structurée et semi-structurée (Boydens, 2001) ainsi qu'un exemple constructif de fonctionnalité adaptée au traitement de ce type d'information.

\subsection{Définition et exemples}

Intuitivement, l'information dite structurée (figure 2) désigne l'information dont le domaine de définition est formellement spécifié a priori par un ensemble de «contraintes d'intégrité »: assertions formelles spécifiant l'ensemble des valeurs

5. «Groupware has been widely promoted as a technology that can facilitate the global integration of geographically distributed organization by overcoming time-space barriers to communication and hence promoting knowledge sharing. Despite huge investments in groupware technologies, however, results have often failed to live up to expectations. While various technical, managerial, and cultural explanations have been proposed, limited consideration has been given to the importance of the social relations that underpin effective communication. » (Kelly et al., 2001). 
admises pour un champ donné (la table contrôlée multilingue de la figure 2 en fournit un exemple).

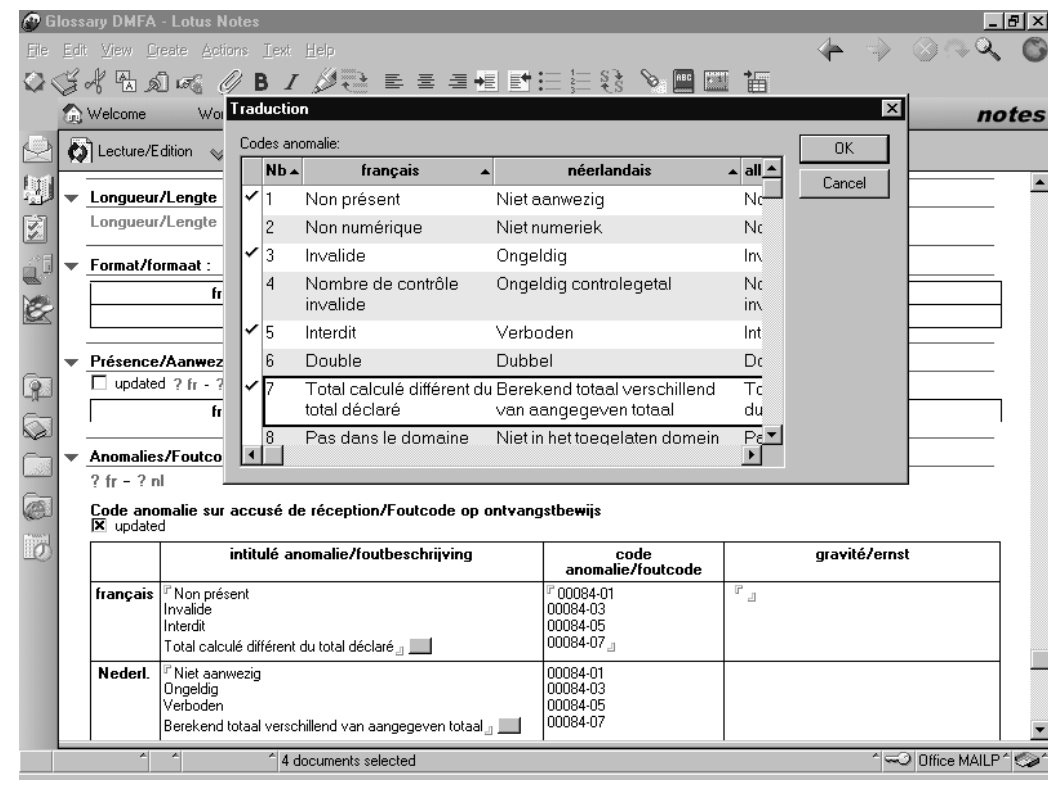

Figure 2. Exemple de champ structuré multilingue

En contraste, l'information dite non structurée désigne l'information textuelle ou multimédia dont le domaine de définition est «libre » (par exemple : collection de lettres ou de comptes rendus de réunions produits via un traitement de texte, collection d'archives numérisées ou non...).

Traditionnellement, le traitement de l'information dite structurée relève de la théorie en matière de conception de bases de données alors que le traitement de l'information dite non structurée relève de la documentologie et de l'automatique documentaire.

Toutefois, la distinction entre les deux branches (traitement de l'information structurée et non structurée) est de plus en plus floue sur les plans conceptuels et logiciels. En effet, plusieurs logiciels de gestion de bases de données (SGBD) actuels ainsi que d'autres systèmes ad hoc (Zaniolo et al., 1997) permettent aujourd'hui la gestion de l'information documentaire d'ordre textuel (Grossman et al., 2001). Par ailleurs, au sein d'un système documentaire, il y a coexistence d'informations structurées (les notices documentaires) et non structurées (les documents textuels et multimédias, par exemple), les premières «documentant » les secondes. Enfin, les collecticiels permettent également de fédérer l'information dite semi-structurée. 
Nous appelons semi-structurées les zones «hybrides» composées de deux champs complémentaires, le premier, structuré, incluant un ensemble de valeurs spécifiées a priori et le second, non structuré, incluant des «valeurs libres». Un exemple de ce type de zone sera présenté au point suivant relatif aux structures d'héritage (figure 4).

La gestion conjointe d'informations structurées, non structurées et semistructurées implique des arbitrages dont il faut prendre conscience. Des difficultés de deux ordres se présentent. D'une part, sur le plan sémantique, le pouvoir contraignant du domaine de définition de champs de différents types est variable : alors que l'on peut vérifier la validité formelle d'un champ structuré, il n'en n'est pas de même à propos d'un champ libre. D'autre part, sur le plan «temporel », alors que certaines valeurs structurées (champs dérivés ou calculés, par exemple) sont mises à jour automatiquement, d'autres (texte libre, par exemple) requièrent une intervention humaine. La maille du temps est donc potentiellement hétérogène au sein d'un même ensemble documentaire. Lorsque l'on consulte l'application à un instant $t$, on se trouve face à la photographie statique de valeurs dont la mise à jour n'est pas nécessairement synchrone.

En contrepartie, un tel environnement offre une grande flexibilité de travail. Par exemple, dans le cas de notre dictionnaire électronique des données, nous avons associé des informations issues d'environnements logiciels hétérogènes en vue de disposer d'une base terminologique «transversale » à plusieurs applications, le tout pouvant être diffusé sous différents formats, comme nous le verrons plus loin. Ainsi, nous avons importé au sein de l'application un thesaurus (Lefèvre, 2000) multilingue («sous forme plate») de plusieurs milliers de termes issus d'une autre application documentaire. L'exploitation de cette source, régulièrement mise à jour, contribue à contrôler les définitions techniques, administratives et juridiques ${ }^{6}$.

\subsection{Les structures d'héritage adaptées à l'information hétérogène}

Nous montrons ici un exemple d'exploitation opérationnelle faisant appel aux différents types d'information présentés au point précédent. Le dictionnaire électronique que nous avons développé est maintenant destiné à gérer les versions documentaires de plusieurs dizaines de bases de données administratives. Ces ensembles documentaires répertorient de nombreux champs communs dont certaines caractéristiques sont identiques (format, par exemple) et d'autres, distinctes (caractère obligatoire ou facultatif d'un champ, par exemple) d'une base à l'autre. Le processus de mise à jour de l'application a été adapté à cette hétérogénéité et inclut

6. On observe par exemple qu'à un même descripteur (loon, en néerlandais) peuvent correspondre plusieurs «équivalents linguistiques» («salaire» et «rémunération», en français, ces deux derniers termes n'ayant pas nécessairement le même sens au niveau juridique). 
maintenant un mécanisme d'héritage partiel dont nous présentons ci-dessous le modèle.

L'héritage partiel se définit comme la relation entre une classe $A$ générique (que nous appelons ici «stéréotype ») et l'ensemble de ses instances $\left\{a_{1}, a_{2}, \ldots a_{n}\right\}$, où les propriétés $\left(\mathrm{p}_{1}, \mathrm{p}_{2}, \ldots \mathrm{p}_{k}\right)$ de la classe $A$ constituent un sous-ensemble des propriétés de chaque objet instantié à partir de la classe $A$ (Meyer, 1997). Lors de l'instantiation, ce sous-ensemble de propriétés génériques peut être complété par un autre sousensemble de propriétés spécifiques à chaque instance $\left(\mathrm{p}_{1+p a l}, \mathrm{p}_{2+p a 2}, \ldots \mathrm{p}_{k+p a n}\right) . \mathrm{Ce}$ mécanisme est applicable à un nombre arbitraire de niveaux « méta ».

Dans notre exemple, les valeurs des propriétés génériques («nom», «domaine de définition », «description », «type », «longueur ») du stéréotype « heure » sont stockées dans une table «contrôlée » de données structurées (figure 3).

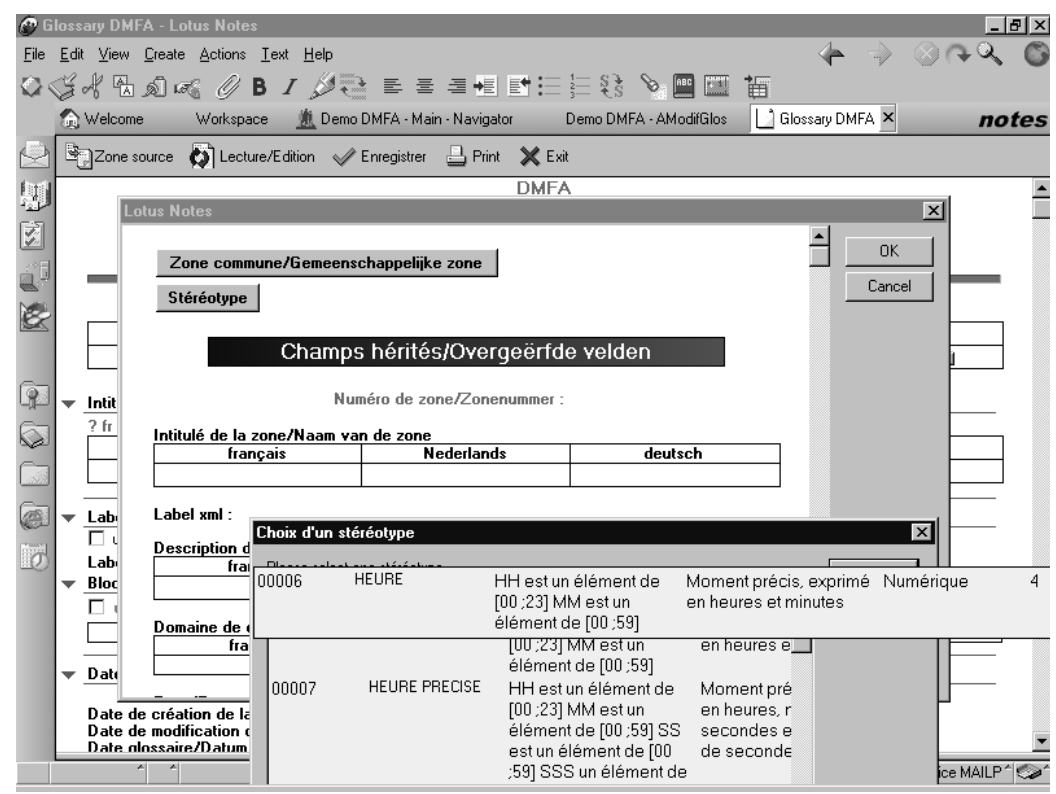

Figure 3. Exemple d'héritage des valeurs d'un concept générique

Ces propriétés génériques multilingues peuvent être héritées dans une fiche documentaire et complétées lors de l'instantiation par des propriétés spécifiques au sein d'un champ semi-structuré (dans l'exemple de la figure 4, en français : «nom » : «heure + de l'intervention médicale de l'hôpital», «domaine de définition»: «Moment précis, exprimé en heures et minutes $+S i$ des soins médicaux ont été administrés par un hôpital suite à l'accident de travail le moment est ici mentionné »...). 


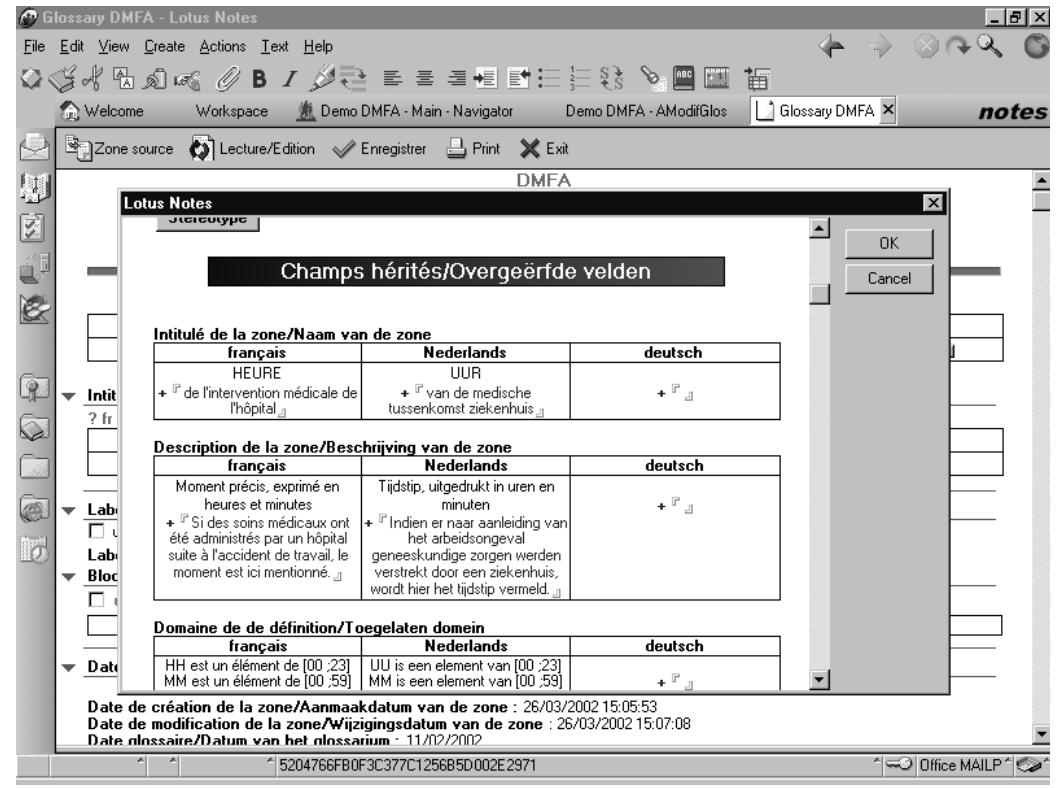

Figure 4. Ajout d'une valeur spécifique à la valeur générique héritée

Les valeurs génériques et spécifiques sont ensuite concaténées en un champ semi-structuré : dans notre exemple, «heure de l'intervention médicale de l'hôpital $»^{7}$. Ces fonctionnalités apportent des avantages en termes de temps de mise à jour (chaque valeur générique structurée correspondant à un «stéréotype » ne devant être encodée qu'une seule fois) et en termes de cohérence. Le système permet d'assurer que les données communes reçoivent les mêmes valeurs et d'éviter les erreurs humaines inhérentes à l'encodage manuel.

Par ailleurs des «vues de gestion» permettent de suivre précisément les mécanismes d'héritage en vigueur pour chaque champ de chaque document à un instant donné. Au niveau de la gestion des versions, dont nous reparlons plus loin, toute modification d'une classe générique («stéréotype ») est propagée dans chaque ensemble de définitions où la valeur de ce champ est héritée.

7. Le stéréotype « heure » peut donner le jour à d'autres instances, via ce système d'héritage : heure de pointage, heure de la signature du formulaire électronique, heure de début de grève/lock-out, etc. 


\section{Aide à l'interprétation de l'information}

Le dictionnaire des données administratives présenté dans cet article s'apparente à un système de méta-information (Boydens, 1996). Le recours à de tels systèmes est stratégique en vue d'interpréter l'information ${ }^{8}$.

\subsection{Les méta-informations : définition et paradoxes}

«Méta-information» est souvent entendu comme «information sur l'information ». Or, à cette acception s'en ajoute une autre : celle de changement. En effet, la particule grecque méta signifie aussi bien l'idée d'un niveau de généralité supérieur (métaphysique, métalangage...) que celle de changement (métabolisme, métastase, métempsycose...). Cette seconde acception est également pertinente en vue de définir un système de «méta-information », lequel implique nécessairement un processus d'interprétation et donc de transformation de l'information documentée. Ainsi, l'interprétation et la description des concepts d'ouvrier et d'employé (exemple de la section 2) peuvent faire évoluer la signification de ces concepts, avec l'évolution de la jurisprudence.

Les systèmes de méta-information comportent trois écueils majeurs. Le premier est lié à ce que ces systèmes sont extensibles à l'infini. Les systèmes de métainformation sont en effet destinés à clarifier l'opacité des codifications formelles et à en réduire l'incertitude. A cette fin, la solution requise réside souvent dans l'utilisation d'une codification plus riche et donc, plus explicite : le langage naturel. Cependant, le langage naturel est son propre métalangage (Boydens, 1999). Dès lors, toute description émise sous cette forme peut faire l'objet d'un nombre infini de commentaires d'ordre supérieur. Ceci se traduit par la lourdeur et le coût de leur gestion pratique, lorsque cette gestion repose sur une mise à jour manuelle. Le second écueil tient à ce que les métadonnées peuvent être elles-mêmes erronées et incertaines: leur validation ne peut faire l'objet systématique de contraintes d'intégrité rigoureuses. Le troisième écueil tient au décalage temporel entre la mise à jour d'une donnée et de la métadonnée correspondante, cette dernière, surtout lorsqu'elle se présente sous une forme textuelle, n'étant généralement créée qu'au terme d'une phase d'analyse plus ou moins longue.

Les systèmes de méta-information sont toutefois incontournables dans certains domaines d'application. Ils sont d'autant plus performants qu'ils sont économes en

8. Ainsi, pendant la guerre du Golfe, environ 28000 des 40000 containers d'armements militaires américains envoyés au Moyen-Orient furent inspectés et inventoriés manuellement tant l'interrogation des bases de données correspondantes, censées en répertorier le contenu, donnait lieu à des résultats incohérents. D'où cette remarque amère : "In general, the physical movement of material is faster than the movement of the supporting information. » (Madnick, 1993). Il va de soi que si chaque base de données avait été correctement documentée et interprétée, l'exploitation automatisée de l'information aurait été plus aisée. 
termes de mise à jour (Imamura et al., 2000) et qu'ils reposent sur un ensemble de sources homogènes, comme nous l'illustrons au point suivant.

\subsection{Génération de l'information et de la méta-information correspondante à partir d'une source unique}

Les applications documentaires administratives incluent non seulement des champs libres mais aussi des listes structurées (codes postaux, catégories d'activité...) qui, dans la pratique, doivent être diffusées à des fins documentaires (dans l'esprit d'un «système de méta-information») mais aussi en vue de tester les déclarations envoyées par les citoyens et stockées dans les bases de données. Afin de rencontrer les deux fonctions, l'application a été conçue dans l'optique du concept WOPM (Write Once Publish Many) de façon à pouvoir générer automatiquement une même table structurée (liste de codes postaux, par exemple) sous différents formats : formats Ascii, XML, Word, Excel et PDF. La même source peut ainsi être utilisée au sein d'applications interdépendantes (figure 5).

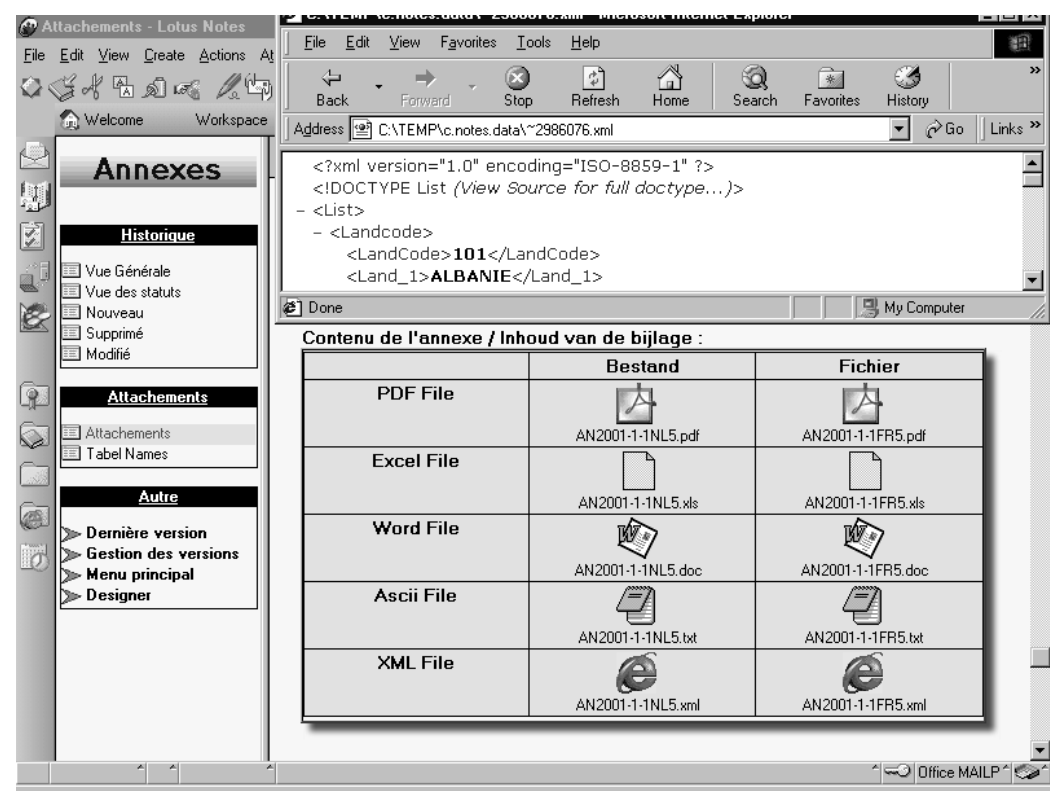

Figure 5. Diffusion de sources communes sous différents formats 


\section{Aide à la construction de l'information}

En anglais, «interpréter » peut se traduire par to construct. De l'interprétation à la construction des concepts, il n'y a qu'un pas. Nous en venons ainsi aux fonctions qui sont au cœur des applications coopératives : la gestion des versions et la mise en place d'un workflow en vue de valider la construction progressive des définitions administratives.

\subsection{La gestion des versions}

Les codes informatiques ne peuvent évoluer que ponctuellement, au terme de mises à jour discrètes, alors que le réel représenté empirique correspondant évolue de façon continue. La prise de conscience de ce mécanisme est fondamentale en vue d'interpréter un système d'information (Boydens, 1999). Dans la pratique, si les écarts entre le réel et sa représentation ne peuvent être comblés, le suivi des versions (Gancarski, 1995) est une fonctionnalité indispensable dans toute application informatique. Dans le dictionnaire présenté ici, chaque mise à jour par rapport à une version antérieure (figure 6) est enregistrée et spécifiée.

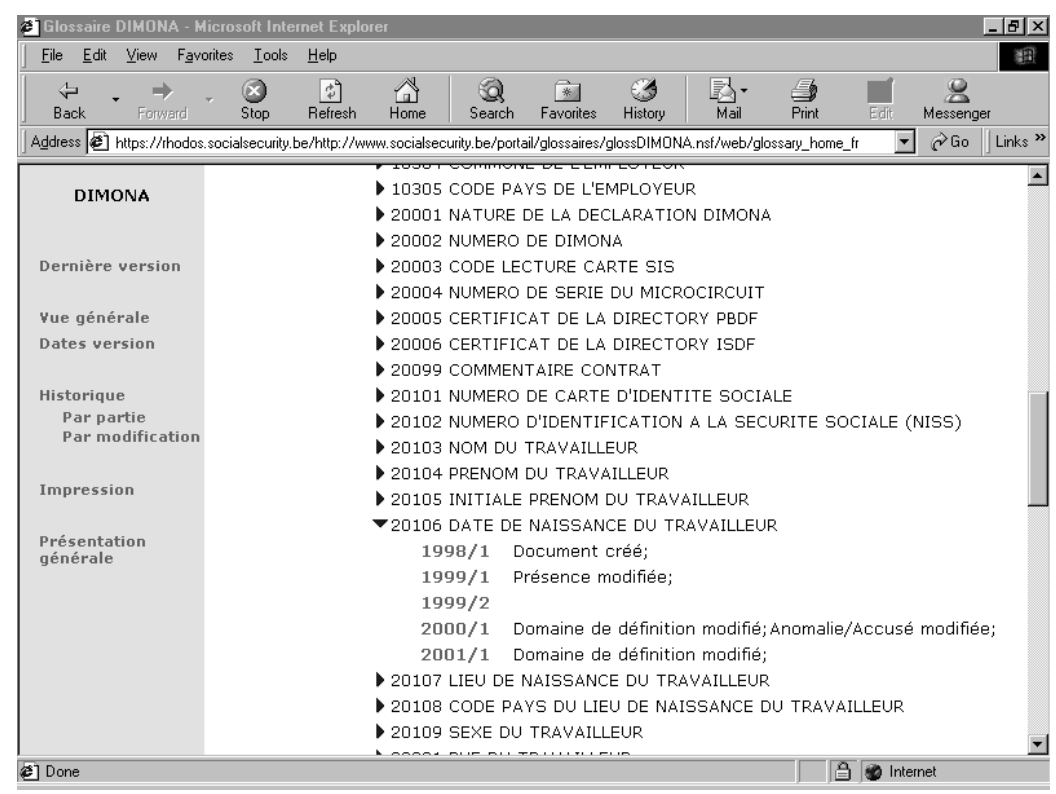

Figure 6. Suivi des versions en consultation (environnement web)

Légalement, l'information administrative doit être conservée durant la période de prescription. Il est dès lors crucial, en vue de traiter des arriérés, par exemple, 
d'identifier précisément les modifications apportées à chaque version documentaire. Chaque item spécifiant la description d'une donnée pour une version considérée renvoie à la fiche correspondante (dans la langue choisie par l'utilisateur) avec la mention du champ modifié par rapport à la version immédiatement antérieure.

\subsection{Les applications coopératives de groupware-workflow comme instrument d'aide à la décision}

Le processus d'interprétation est potentiellement infini; dans la pratique, il requiert un arrêt, une prise de décision humaine. En vue d'accompagner cette prise de décision dans le cadre de la constitution d'une nouvelle version documentaire, un système de workflow guide le déploiement du dictionnaire électronique. Le responsable d'un ensemble documentaire donné a la possibilité de définir les interlocuteurs qui interviendront dans les différentes phases présidant à la mise en production : secrétariat, vérification, traduction, «institution responsable », etc. Par ailleurs, lors de la création de chaque nouvelle version, l'historique des échanges entre les différents responsables est conservé, de façon à garder un suivi du processus d'interprétation.

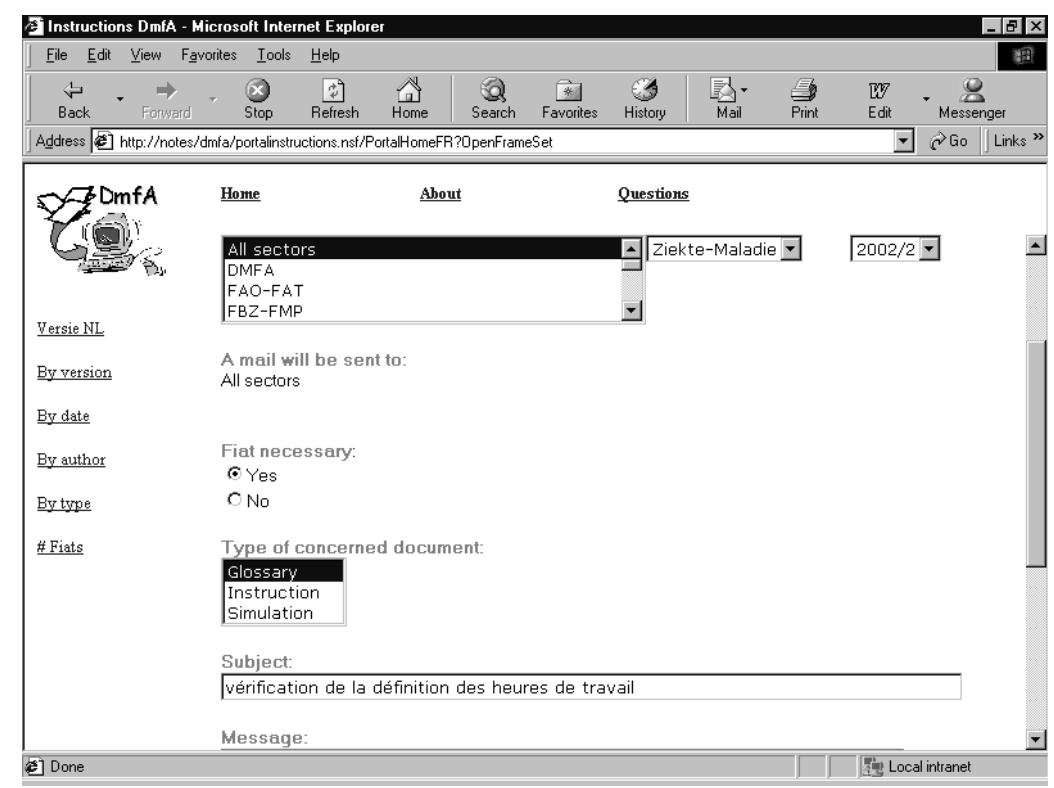

Figure 7. Workflow de validation : exemple d'écran dans un environnement web

Comme l'illustre la figure 7 , à un stade donné du processus de validation, le responsable en charge d'un type de document, indique, pour une version 
documentaire, quels sont les acteurs concernés par une procédure donnée (lesquels recevront un «mail d'alerte » en vue d'une validation) et si un fiat (accord) officiel est requis. Les procédures de validation sont limitées aux institutions membres de l'extranet de la sécurité sociale, responsables de la documentation administrative. Une vue permet au gestionnaire de suivre le nombre de fiats requis pour la publication d'une version (des procédures de décision sont prévues en cas de «nonréponse » de l'un des acteurs dans les délais fixés).

\section{Conclusions et perspectives de recherche ultérieure}

Les modèles de bases de données reposent formellement sur le principe de l'identité, de la non contradiction et du tiers exclu. En l'absence de modification exogène, $A$ est $A$ et le restera toujours. Par contre, dans le cadre d'un système d'information empirique, un objet n'est jamais nécessairement identique à lui-même. Nous entendons par là, d'une part, qu'à un même concept empirique peut correspondre à un instant donné une pluralité de significations interagissantes et, d'autre part, qu'interagissant, ces significations sont évolutives. C'est toute la question de l'instabilité des définitions administratives évoquée au seuil de cet article (section 2). Face à cette question, les applications documentaires coopératives de type groupware-workflow offrent des environnements souples en vue d'accompagner l'évolution des définitions, bien que, nous l'avons vu, cette souplesse implique certaines difficultés méthodologiques et conceptuelles (section 2.3.). Le dictionnaire électronique des données dont nous avons décrit succinctement les principales fonctionnalités permet la gestion conjointe d'informations structurées, non structurées et semi-structurées. Au sein de cet environnement, il est possible de développer des structures d'héritage correspondant à la nature hétérogène de l'information traitée (section 3). Ces fonctionnalités contribuent à garantir l'intégrité de l'information. En tant que système de métainformation, l'application offre la possibilité d'exploiter une même source (une table de paramètres, par exemple) à des fins distinctes et dans différents formats, de façon à éviter les incohérences lors des traitements (section 4). Enfin, en tant qu'instrument de gestion coopérative, le dictionnaire électronique offre des fonctions avancées de gestion des versions et de workflow, de façon à guider le processus de construction de l'information (section 5).

L'application dont nous avons présenté les fonctionnalités est en production dans un environnement web. Un des éléments les plus déterminants en vue d'en garantir l'efficacité réside dans une prise en compte continue du facteur humain et dans la mise en œuvre de procédures d'exploitation rigoureuses validées par tous. A cette fin, une cellule technique, dédiée exclusivement à l'administration du workflow documentaire associé à l'application, a été mise en place afin de veiller au maintien de l'intégrité de l'ensemble et au respect des procédures de mise à jour. 
Dans une phase ultérieure, nous prévoyons d'étendre le dictionnaire électronique présenté ici dans une application plus vaste, reliant conceptuellement les définitions législatives, administratives et techniques : des aides contextuelles ciblées pourraient être incorporées, dans un environnement web, aux formulaires électroniques correspondants. Le traitement conjoint de ces différents types de définition est toutefois complexe. A un instant $t$, les relations entre chacun de ces types de définition ne sont ni biunivoques ni monohiérarchiques. En effet, chaque niveau sémantique évolue à un rythme propre tout en interagissant (Boydens, 1999) : l'évolution de la législation contraint les définitions techniques mais en retour, les codifications formelles d'une loi ont une incidence sur son application pratique et, de là, sur son évolution future.

\section{Remerciements}

L'auteur tient à remercier l'équipe du projet Instructions de la SmalS-MvM pour sa contribution active à la conception et au développement de l'application présentée dans cet article.

\section{Bibliographie}

Bachelard G., Essai sur la connaissance approchée, Paris, Vrin, 1981.

Boucourechliev J., «L'informatique face à la complexification du droit: facteur positif, négatif... ou pervers », Droit et informatique. L'hermine et la puce, Collection $F$. R. BULL, Paris, Masson, 1992, p. 39-48.

Boydens I., «Les systèmes de méta-information, instruments d'interprétation critique des sources informatiques », History and Computing, vol. 1, n 8, janvier 1996, p. 1123.

Boydens I., Pirotte A. et Zimanyi E., «Managing Constraints Violations in Administrative Information Systems », Proceedings of the 7th IFIP 2.6 Conference on Data Semantics, DS-7, Leysin, octobre 1997, Chapman \& Hall, p. 241-264.

Boydens I., Informatique, normes et temps, Bruxelles, Editions E. Bruylant, 1999.

Boydens I., Introduction à la documentologie (syllabus), Bruxelles, Presses de l'Université Libre de Bruxelles, 1 ère édition 2000-01/1.

Chen P. P., «The Entity-Relationship Model: Toward a Unified View of Data », ACM Transactions on Database Systems, vol. 1, n 1, 1977, p. 9-37.

Delteil A., Faron C. et Dieng R., «Extending RDF(S) with Contextual and Definitional Knowledge », Proceedings of the UCAI-01 Workshop «E-Business and the Intelligent Web », Seattle, 5 août 2001, http://www.csd.abdn.ac.uk/ebiweb/programme.html

Gancarski S. et Jomier G., «Gestion des versions d'entités et de leurs contextes : analyse et perspectives », Ingéniérie des systèmes d'information, vol. 3, n ${ }^{\circ}$ 6, 1995, p. 677-711. 
Gray P., Hui K. et Preece A., «An Expressive Constraint Language for Semantic Web Applications », Proceedings of the UCAI-01 Workshop "E-Business and the Intelligent Web », Seattle, 5 août 2001, http://www.csd.abdn.ac.uk/ebiweb/programme.html

Grossman D. et Frieder O., «Integrating Structured Data and Text. Part 2 », Intelligent Enterprise, vol. 4, $\mathrm{n}^{\circ}$ 16, 24 octobre 2001, p. 20-23.

Imamura M., Nagahama R., Suzuki K., Watabe A. et Tsuji H., "Metadata Represantation in XML for Internet-Based Elextronic XML Application from Business to Governement", Proceedings of the Seventh International Conference on Parallel and Distributed Systems Workshops ICPADS'00, 4-7 juillet 2000, Iwate, Los Alamitos, IEEE Computer Society, p. 387-392.

Kelly S. et Jones M., "Groupware and the social infrastructure of communication ", Communications of the ACM, vol. 44, $\mathrm{n}^{\circ}$ 12, décembre 2001, p. 77-79.

Lefèvre P., La recherche d'informations. Du texte intégral au thesaurus, Paris, Editions Hermès, 2000.

Madnick S. E., «The Voice of the Customer : Innovative and Useful Research Directions». In Agrawal R., Baker S., et Bell D., éds, Proceedings of the 19th Conference on Very Large Databases, Dublin, VLDB, 1993, p. 702.

Mcllraith S. A., Son T. C. et Zeng H., «Mobilizing the Semantic Web with DAML-Enabled Web Services », Proceedings of the UCAI-O1 Workshop "E-Business and the Intelligent Web», Seattle, 5 août 2001, http://www.csd.abdn.ac.uk/ebiweb/programme.html

Meyer B., Object Oriented Software Construction, New Jersey, Prentice Hall, 1997.

Osguthorpe G., Steele B. et Houlderoft A., «A Method for Developping CSCW Systems », Proceedings ot the $5^{\text {th }}$ International Workshops on Enabling Technologies Infrastructures for Collaborative Enterprises (WET ICE'96), Stanford, 19-21 juin 1996, Los Alamitos, IEEE Computer Society, 1996, p. 214-219.

Phelps T. A. et Wilensky R., «Multivalent Documents », Communcations of the ACM, vol. $43, \mathrm{n}^{\circ} 1$, juin 2000 , p. 83-90.

Qu, C., Engel, T., Meinel, C., «Implementation of an Enterprise-Level Groupware System Based on J2EE Platform and WebDAV Protocol», Proceedings of the Fourth International Enterprise Distributed Object Computing Conference (EDOC'2000), Makuhari, 28-28 septembre 2000, Los Alamitos, IEEE Computer Society, 2000, p. 160169.

Thomasset C., Bourcier D., éds, Interpréter le droit: le sens, l'interprète, la machine, Bruxelles, Bruylant, 1997.

Zaniolo C., Ceri S., Faloutsos C., Snodgrass R. T., Subrahmanian V. S. et Zicari R., Advanced Database Systems, San Francisco, Morgan Kaufmann Publishers, 1997. 